\title{
CCL19-induced chemokine receptor 7 activates the phosphoinositide-3 kinase-mediated invasive pathway through Cdc42 in metastatic squamous cell carcinoma of the head and neck
}

\author{
ZHEN-JIN ZHAO ${ }^{1}$, FA-YU LIU ${ }^{2,3}$, PENG LI ${ }^{2,3}$, XUE DING $^{2,3}$, ZHI-HONG ZONG ${ }^{4}$ and CHANG-FU SUN ${ }^{2,3}$ \\ ${ }^{1}$ Department of Orthodontics; ${ }^{2}$ Department of Oromaxillofacial-Head and Neck Surgery; ${ }^{3}$ Department of Oral Maxillofacial \\ Surgery; School of Stomatology, China Medical University, No. 117. Nanjing Bei Street, Heping District, Shenyang, \\ Liaoning 110002; ${ }^{4}$ Department of Biochemistry and Molecular Biology, China Medical University, No. 92, \\ Beier Road, Heping District, Shenyang, Liaoning 110001, P.R. China
}

Received September 21, 2010; Accepted October 26, 2010

DOI: 10.3892/or.2010.1109

\begin{abstract}
Metastatic squamous cell carcinoma of the head and neck (SCCHN) has been shown to express chemokine receptor 7 (CCR7), which activates phosphoinositide-3 kinase (PI3K) to promote invasion and survival of SCCHN cells. We hypothesized that $\mathrm{Cdc} 42$ might be involved in the CCR7-PI3K pathway. Adhesion assays, migration assays, immunofluorescence staining, Western blotting and immunohistochemical analysis were used to find whether $\mathrm{Cdc} 42$ can be activated by CCL19 (the CCR7 ligand) and its role in SCCHN. Results showed that CCL19 induced polarized localization of Cdc42 and actin polymerization in the leading edge of migrating cells. The level of activated membranebound Cdc42 was elevated, as measured by the GTPase activity pull-down assay. The increased membrane localization and membrane-bound activity of $\mathrm{Cdc} 42$ were abolished by CCR7 and PI3K inhibition, indicating the involvement of Cdc42 in the CCR7-PI3K cascade. Knockdown of Cdc42 by small interfering RNA (siRNA) led to significant reduction in the activation of Rac, filamentous actin (F-actin) accumulation as well as in the migration and invasion induced by CCL19. Taken together, our data indicate the important role played by $\mathrm{Cdc} 42$ in CCL19-induced migration and invasion of SCCHN cells.
\end{abstract}

Correspondence to: Dr Chang-Fu Sun, Department of Oromaxillofacial-Head and Neck Surgery, School of Stomatology, China Medical University, No. 117. Nanjing Bei Street, Heping District, Shenyang, Liaoning 110002, P.R. China

E-mail: changfusun@hotmail.com

Key words: chemokine receptor 7, squamous cell carcinoma of head and neck, migration, invasion, $\mathrm{Cdc} 42$, phosphoinositide- 3 kinase, signal transduction, actin cytoskeleton

\section{Introduction}

Survival for patients with squamous cell carcinoma of head and neck (SCCHN) is only $30-40 \%$, mainly due to the frequent presence of metastasis at diagnosis (1). Metastasis is the result of several sequential steps and represents a highly organized, non-random and organ-selective process (2). The mechanisms determining the directional migration and invasion of SCCHN cells into specific organs remain to be established.

Chemokines are a superfamily of small, cytokine-like proteins that induce cytoskeleton rearrangement, firm adhesion to endothelial cells and directional migration (3). They are involved in all stages of tumor development, including initiation, growth and progression (4). Recently, metastatic SCCHN cells were shown to express chemokine receptor 7 (CCR7), which activated phosphoinositide-3 kinase (PI3K)mediated invasive and survival pathway independent of EGFR (5-9).

Chemotaxis, a process that cells interpret shallow gradients of external attractant to produce directed cell migration (10), allows leukocytes to crawl to sites of infection and inflammation, fibroblasts to enter a wound, amoebae of Dictyostelium discoideum to form multicellular organisms, and budding yeast cells to find mating partners (11). It is also believed to be one of the mechanisms underlying tumor metastasis (4). The cell cytoskeleton is crucially involved in the regulation of chemotaxis and other motile-related functions (12). Small GTPases of the Rho family, including Rac and Cdc42, could promote formation of actin polymer and play a key role in actin cytoskeletal rearrangement during cell migration $(13,14)$. CCR7 was shown to induce cell motility though PI3K but blockage of Akt/PKB could not reduce the cell motility (6), indicating the involvement of other downstream signaling molecules in the process. Recently, it was demonstrated that activation of Rac or $\mathrm{Cdc} 42$ or both by $\mathrm{Gi}$ proteins were involved in dendritic cells (DC) $(13,15)$ and mouse fibroblast (16). But no reports have demonstrated the activation of Cdc42 and Rac by chemokine ligand 19 (CCL19) in SCCHN 
cells. Therefore, we hypothesized that Cdc42 might be involved in CCR7-PI3K cascade during invasion of SCCHN cells. Our results showed that CCL19 induced the activation and polarized membrane localization of $\mathrm{Cdc} 42$, which were associated with polarity of actin polymerization in the leading edge of migrating cells. Cdc42 blockage by Toxin B and Cdc42 siRNA could diminish the activation of Rac, polarity of filamentous actin (F-actin), and thus motility of SCCHN cells.

\section{Materials and methods}

Cell lines and culture conditions. SCCHN cell lines PCI-4B and PCI-37B were kindly donated from University of Pittsburgh Cancer Institute, which have been well characterized (5). The cells were cultured in DMEM medium (Invitrogen Carlsbad, CA, USA) containing 10\% fetal bovine serum (Gibco-BRL Corp., Grand Island, NY, USA), 100 U/ml penicillin $\mathrm{G}$ and $100 \mu \mathrm{g} / \mathrm{ml}$ streptomycin (Invitrogen). When inhibitors were used, inhibitor treatment at the dose used did not affect the viability or expression of CCR7 by the cells.

Antibodies and reagents. The CCR7 chemokine ligand, CCL19 (MIP-3ß) was purchased from R\&D System (Minneapolis, MN, USA). The PI3K inhibitor LY294002 was puchased from Calbiochem (San Diego, CA, USA). Mouse anti-CCR7 antibody was from BD Biosciences (San Jose, CA, USA). Toxin B and TRITC-labelled phalloidin were from Sigma (Sigma-Aldrich, St. Louis, MO, USA). Goat polyclone Cdc42 anti-body (C-20), rabbit polyclone Cdc42 antibody (P1), goat polyclone Rac antibody (S-18) rabbit polyclone Rac antibody (C-11), siRNA targeting human CCR7 and Cdc42, and non-specific control siRNA were from Santa Cruz Biotechnology (Santa Cruz, CA, USA). Lipofectamine ${ }^{\mathrm{TM}}$ 2000 was purchased from Invitrogen. GST-PBD vector was a kind gift of Dr M.A. Schwartz (University of Virginia, Charlottesville, VA, USA).

RNA interference of $C d c 42$. Non-specific control siRNA or Cdc42 siRNA (100 pmol) were transfected into SCCHN cells using Lipofectamine ${ }^{\mathrm{TM}} 2000$ according to the manufacturer's instructions. The cells were harvested for analysis $48 \mathrm{~h}$ after transfection.

Chemotaxis assay. Cell migration studies were performed as described previously (6). Briefly, disposable 24-well transwell inserts (Corning) with $8 \mu \mathrm{m}$ pore size were run in triplicate in DMEM with $0.5 \%(\mathrm{w} / \mathrm{v})$ BSA (Invitrogen). Aliquots of the chemokine CCL19 were added to the lower chamber at a concentration of $500 \mathrm{ng} / \mathrm{ml}$. PCI-4B and -37B cell suspensions $\left(2 \times 10^{5}\right)$, which were pretreated with/without CCR7 mAb $(10 \mu \mathrm{g} / \mathrm{ml})$, PI3K inhibitor LY294002 $(10 \mu \mathrm{M})$, Toxin B $(100 \mathrm{pg} / \mathrm{ml})$ for $4 \mathrm{~h}$ and CCR7 siRNA (24 h) (100 pmol, $48 \mathrm{~h})$ were placed in the top of inserts. After $4 \mathrm{~h}$ of incubation, the cells on the upper surface of inserts were removed with a cell harvester, and the filter was washed with medium. A $50 \mu 1$ aliquot of trypsin-EDTA (Invitrogen Corp.) was added to the filter for $3 \mathrm{~min}$ at $37^{\circ} \mathrm{C}, 5 \% \mathrm{CO}_{2}$ before centrifuging at $1500 \mathrm{rpm}$ for $10 \mathrm{~min}$. The filter was carefully removed, and cells in each lower well were counted under a light micro- scope in at least five different fields (original magnification, $\mathrm{x} 200$ ). Mean \pm standard deviation (SD) was recorded for each condition and chemotaxis index was calculated based on the control, random migration.

Matrigel invasion assay. Cell invasion was quantified in vitro using Matrigel-coated semipermeable, modified inserts with a pore size of $8 \mu \mathrm{m}$. The analysis of Matrigel invasion assay was performed as described in the chemotaxis assay incubated with CC19 for $36 \mathrm{~h}$. Invasion was normalized to the nonspecific cell invasion (from media pulsed wells).

Scrap wound-healing assay. SCCHN cells were plated in a 24-well plate at initial density of $1.5 \times 10^{5} \mathrm{cells} / \mathrm{cm}^{2}$. A uniform monolayer formed in 2-3 days. All wounding assays were performed in a serum-free medium. Cells were pretreated with/ without CCR7 mAb $(10 \mu \mathrm{g} / \mathrm{ml})$, PI3K inhibitor LY294002 $(10 \mu \mathrm{M})$ and Toxin $\mathrm{B}(100 \mathrm{pg} / \mathrm{ml})$ for $4 \mathrm{~h}$, or transfected with Cdc42 siRNA for $48 \mathrm{~h}$ before the start of the experiments. A micropipette tip was used to create a wound in the monolayer by scrapping. Then cells were incubation with CCL19 (500 ng/ml, 0.5 h).

Actin polymerization assay. PCI-4B and $-37 \mathrm{~B}$ cells were pretreated with/without CCR7 mAb, LY294002 and Toxin B or transfected with Cdc42 siRNA for $48 \mathrm{~h}$ before scrapping. After incubation with CCL19 (500 ng/ml) for $0.5 \mathrm{~h}$, cells were fixed, permeabilized and stained with TRITC-labeled phalloidin. Following labeling, the samples were washed 3 times for $10 \mathrm{~min}$ each in PBS to remove the unincorporated label. F-actin distribution following CCL19 stimulation was evaluated by confocal laser scanning microscope (CLSM, Leica SP2, Germany).

Cdc42 staining. PCI-37B cells were pretreated as described in scrap wound-healing assay. After incubation of CCL19 $(500 \mathrm{ng} / \mathrm{ml}, 0.5 \mathrm{~h})$, the cells were fixed, permeabilized and blocked with $10 \%$ horse serum and $3 \%$ BSA in PBS. The cells were then incubated in rabbit anti-Cdc42 polyclonal antibody (diluted at 1:50) overnight at $4^{\circ} \mathrm{C}$, followed by $1 \mathrm{~h}$ incubation with FITC-labeled goat anti-rabbit IgG (diluted as 1:100). Between each steps described above, the cells were washed with PBS three times. The localization of $\mathrm{Cdc} 42$ was evaluated by CLSM.

Cdc42/Rac GTPase assay. GST-PBD vector was used to produce recombinant GST fused to the Rac/Cdc42-binding domain of PAK in E. coli. The recombinant GST fused to the Rac/Cdc42-binding domain of PAK could specifically binds to and precipitates cdc42-GTP from cell lysates. PCI-37B cells were pretreated with/without CCR7 mAb $(10 \mu \mathrm{g} / \mathrm{ml})$, PI3K inhibitor LY294002 $(10 \mu \mathrm{M})$ and Toxin B (100 pg/ml) for $4 \mathrm{~h}$ prior to incubation with CCL19 at concentration of $500 \mathrm{ng} / \mathrm{ml}$ for $0.5 \mathrm{~h}$. Cells were chilled on ice, washed with ice-cold PBS, and lysed in buffer containing $40 \mathrm{mM}$ Trisbase, $5 \mathrm{M}$ urea, $2 \mathrm{M}$ thiourea, 2\% CHAPS, 2\% SB 3-10, $5 \mathrm{mM}$ TCEP or $10 \mathrm{mM}$ DTT, protease inhibitors $(5 \mathrm{mM}$ benzamidine, $5 \mathrm{mM}$ PMSF, $1 \mathrm{mM}$ N-ethylamide). Cell lysate was incubated with $20 \mu \mathrm{g}$ of recombinant GST fused to the Rac/Cdc42binding domain of PAK overnight followed by incubation 
Table I. Correlation between CCR7, Cdc42 expression and clinicopathological factors of SCCHN.

\begin{tabular}{|c|c|c|c|c|c|c|c|}
\hline \multirow{2}{*}{$\begin{array}{l}\text { Clinicopathological } \\
\text { characteristics }\end{array}$} & \multirow[b]{2}{*}{ No. of cases } & \multicolumn{2}{|c|}{ CCR7 } & \multirow[b]{2}{*}{$\chi^{2}$} & \multicolumn{2}{|c|}{$\mathrm{Cdc} 42$} & \multirow[b]{2}{*}{$\chi^{2}$} \\
\hline & & +-+++ & - & & +-+++ & - & \\
\hline \multicolumn{8}{|l|}{ Age } \\
\hline$\geq 60$ & 40 & 25 & 15 & 0.032 & 22 & 18 & 0.844 \\
\hline$<60$ & 38 & 23 & 15 & & 18 & 20 & \\
\hline \multicolumn{8}{|l|}{ Gender } \\
\hline Male & 50 & 32 & 18 & 0.357 & 24 & 26 & 3.344 \\
\hline Female & 28 & 16 & 12 & & 9 & 19 & \\
\hline \multicolumn{8}{|l|}{ Tumor size } \\
\hline $\mathrm{T} 1, \mathrm{~T} 2$ & 65 & 37 & 28 & 3.510 & 25 & 40 & $9.286^{\mathrm{a}}$ \\
\hline $\mathrm{T} 3, \mathrm{~T} 4$ & 13 & 11 & 2 & & 10 & 3 & \\
\hline \multicolumn{8}{|l|}{ Clinical stage } \\
\hline I, II & 37 & 15 & 22 & $13.113^{\mathrm{a}}$ & 7 & 30 & $17.046^{\mathrm{a}}$ \\
\hline III, IV & 41 & 33 & 8 & & 28 & 13 & \\
\hline \multicolumn{8}{|l|}{ Nodal metastasis } \\
\hline Yes & 37 & 29 & 8 & $8.434^{\mathrm{a}}$ & 24 & 13 & $27.391^{\mathrm{a}}$ \\
\hline No & 41 & 19 & 22 & & 8 & 33 & \\
\hline
\end{tabular}

${ }^{a} \mathrm{P}<0.05$ (the internal difference of CCR7 or Cdc42 expression within clinicopathological characteristics).

with glutathione-agarose beads (Pharmacia, Peapack, NJ) for $4 \mathrm{~h}$ at $4^{\circ} \mathrm{C}$. The beads were spun down and washed with lysis buffer 3 times. The bound proteins were eluted with SDS sample buffer and analyzed by Western blotting using a rabbit polyclone Cdc42 antibody (P1) or goat polyclone Rac antibody (S-18). Whole cell lysates were also analyzed using goat polyclone $\mathrm{Cdc} 42$ antibody (C-20) or rabbit polyclone Rac antibody (C-11) for normalization.

Immunohistochemical analysis. Seventy-eight specimens of SCCHN tumors with the adjacent metastatic (or normal) lymph nodes and 10 specimens of normal human oral mucosal tissue were obtained from Head and Neck Tumor Center, School of Stomatology, China Medical University. All the specimens were obtained with the consent of the patients before surgery and in accordance with Health Insurance Portability. The classification of SCCHN, including primary tumors $(\mathrm{T})$, regional lymph nodes $(\mathrm{N})$, distant metastasis (M) and stage grouping, was determined according to the rules of the International Union Against Cancer (UICC) for Head and Neck Cancer (tumor node metastasis, TNM classification, 1997). Immunohistochemical staining used conventional horseradish peroxidase immunohistochemical staining methods. In brief, 5- $\mu \mathrm{m}$ sections of the specimens were deparaffinized and hydrated with $0.6 \% \mathrm{H}_{2} \mathrm{O}_{2}$ in methanol to inhibit endogenous peroxidase, performed antigen retrieval and incubated with normal blocking serum for $10 \mathrm{~min}$. Then the sections were incubated with primary antibodies (1:100): CCR7-specific monoclonal antibody and rabbit anti-Cdc42 polyclonal antibody overnight at $4{ }^{\circ} \mathrm{C}$. Immunodetection was performed using peroxidase labeled secondary antibody (R\&D System) and diaminobenzidine for visualization. All the sections were counterstained with hematoxylin (Sigma). Negative controls included omission of the primary antibody. The cell morphology was analyzed by microscopy (Nikon Eclipse 80i, Tokyo, Japan) at x100-400 magnification. According to the percentage of positive tumor cells, all these cells were scored as negative $(-),<10 \%$ or no staining; weak positive (+), 11-50\%; positive $(++), 51-75 \%$; or strongly positive $(+++),>75 \%$.

Statistical analysis. All experiments were run in triplicate and repeated at least three times. Numerical data were expressed as mean \pm standard deviation (SD). Statistical differences between groups were evaluated using standard analysis of variance (ANOVA). Values of $\mathrm{P}<0.05$ were considered significant. Test for violations of equal variance assumption was performed by using the Levene test. All pairwise comparisons between means were made using SNK (Student-NeumanKeuls) test at an $\alpha$ of 0.05 . All statistical analysis were performed with the software SPSS 13.0.

\section{Results}

Cdc42 had significantly higher expression in tumor tissues and metastatic lymph nodes. By immunohistochemistry, we investigated the expression of CCR7 and Cdc42 in SCCHN tumor tissues, metastatic lymph nodes, normal lymph nodes and oral mucosal tissues. CCR7 and $\mathrm{Cdc} 42$ were both found in the cell membrane and cytoplasm, mainly expressed in the 


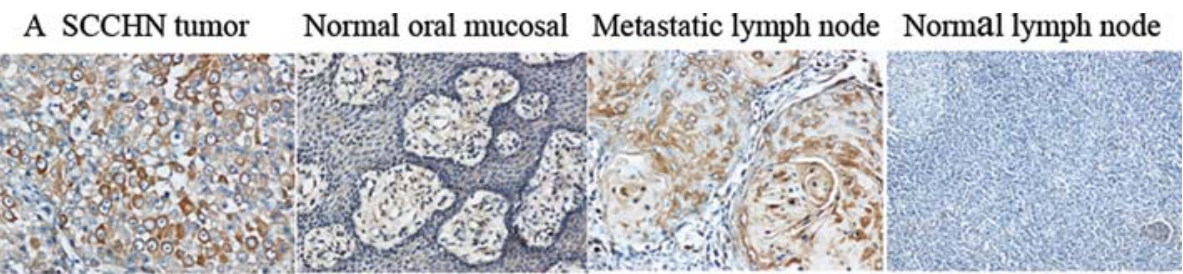

B SCCHN tumor Normal oral mucosal Metastatic lymph node Normal lymph node

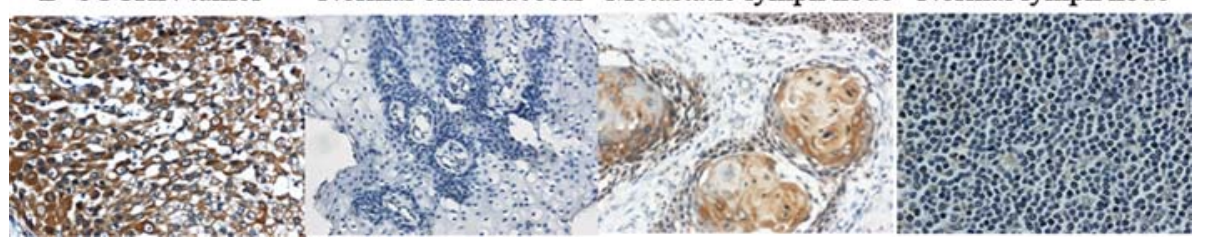

Figure 1. Immunohistochemical staining of CCR7 and Cdc42 in normal tissues, primary SCCHN and metastatic lymph nodes. SCCHN tumor tissues, metastatic lymph nodes, normal lymph nodes and 10 normal human oral mucosal tissues [original magnification (o.m.), x200] show immunoreactivity for CCR7 (A) and Cdc42 (B). CCR7 immunoreactivity was observed mainly in the cell membrane and cytoplasm of tumor cells, conforming with Cdc42 immunoreactivity. CCR7 and Cdc42 expressed in normal lymph nodes and normal human oral mucosal tissues were weak or absent.

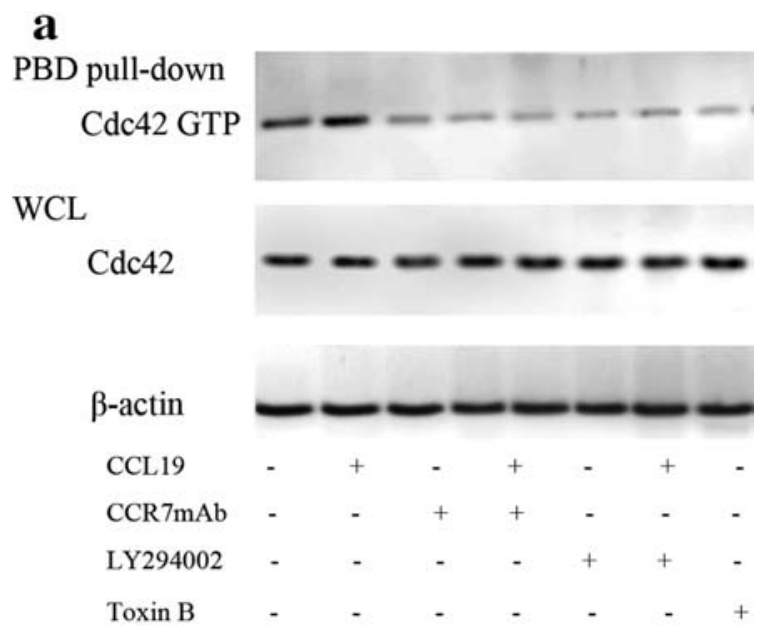

b
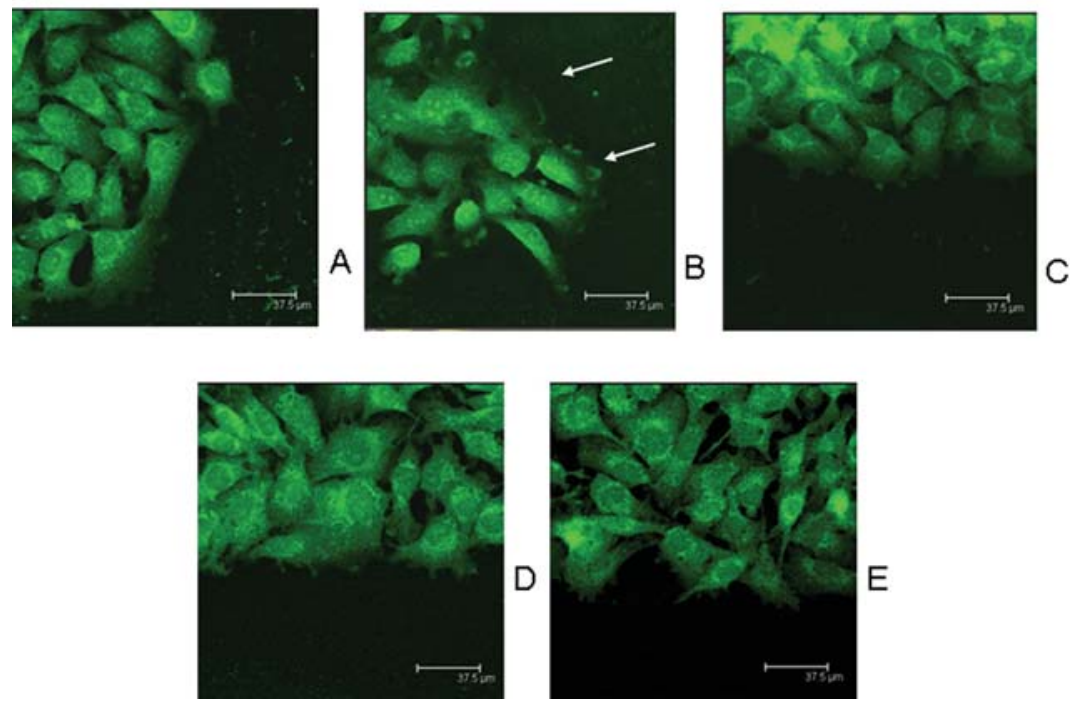

Figure 2. CCL19-induced membrane-bound Cdc42 activation and polarized membrane localization in PCI-37B. (a) In PCI-37B, Cdc42 activated by CCL19 $(500 \mathrm{ng} / \mathrm{ml})$ stimulation for $0.5 \mathrm{~h}$ and the activation were blocked by pretreatment with CCR7 mAb, PI3K inhibitor LY294002 and Toxin B. Levels of total Cdc42 were determined for whole cell lysates (WCL) by Western blotting. Western blot analysis of B-actin was done to confirm equal protein loading. Levels of membrane-bound activated Cdc42 were determined using a GTPase activity pull-down assay (PBD pull-down) followed by Western blotting. GST-p21PAK was used for GST pull-down of activated Cdc42. (b) In PCI-37B, treatment with CCL19 induced significant membrane localization of Cdc42 in the leading edge of PCI-37B that was located along the wound (arrow). The change was blocked by pretreatment with inhibitors. (A), control; (B), CCL19; (C), pretreated with CCR7mAb and incubated with CCL19; (D), pretreated with LY294002 and incubated with CCL19; (E), pretreated with Toxin B and incubated with CCL19. Cdc42 was stained with immunofluorescence and visualized using confocal laser scanning microscope (CLSM). 
surrounding of stroma in tumors cells and metastatic lymph node cells. The number of stained cells was low or absence in normal lymph nodes and oral mucosal tissues (Fig. 1 and Table I). The expression of CCR7 and Cdc42 were both significantly correlated with cervical lymph node metastasis and SCCHN clinical stage $(\mathrm{P}<0.05)$. However, there were no significant differences between CCR7 or Cdc42 expression and age or gender $(\mathrm{P}>0.05)$. A moderate correlation was observed between CCR7 and Cdc42 expression in SCCHN tumor tissues $(\mathrm{P}<0.05)$ and metastatic lymph nodes $(\mathrm{P}<0.05)$, but there was no significant correlation between normal lymph nodes $(\mathrm{P}>0.05)$ and normal oral mucosal tissues $(\mathrm{P}>0.05)$.

CCL19-induced membrane-bound Cdc42 activation and polarized membrane localization in PIC-37B. To test whether CCL19 treatment could activate membrane-bound Cdc42 in SCCHN cell line PCI-37B, we first examined the level of membrane-bound activated Cdc42 (GTP-bound form) by performing the GTPase pull-down assay using recombinant GTPase-binding domain derived from the p21-activated kinase-1, a typical substrate of $\mathrm{Cdc} 42$ and Rac. Although the levels of membrane-bound $\mathrm{Cdc} 42$ remained unchanged, the levels of membrane-bound activated $\mathrm{Cdc} 42$ were found to increase significantly $0.5 \mathrm{~h}$ after CCL19 treatment compared with control group ( $\mathrm{P}=0.003)$, whereas CCR7mAb, LY294002 and Toxin B could partly inhibit CCL19-induced activation of membrane-bound Cdc42 compared with CCL19-stimulation group $(\mathrm{P}<0.01)$ (Fig. 2a). These results indicate that CCL19 treatment might lead to activation of Cdc42 in SCCHN cells via the CCR7-PI3K pathway.

Rho GTPase function is critically dependent on association of the GTPases with membranes and their subcellular localization, activated GTPases need to translocate to the cell membrane, where they bind and activate their downstream effectors $(17,18)$. To determine whether CCL19 affected the cellular localization of $\mathrm{Cdc} 42$, we performed $\mathrm{Cdc} 42$ immunofluorescence staining on the SCCHN cell line PCI$37 \mathrm{~B}$ in the scratch wound-healing assay. Treatment with CCL19 induced significant membrane localization of Cdc42 in the leading edge of cells that were located along the wound (Fig. 2b). In contrast, pretreatment with CCR7mAb, LY294002 or Toxin B significantly reduced the CCL19induced polarized membrane localization of $\mathrm{Cdc} 42$. Together, the above results indicate that CCR7-PI3K pathway is required for activation and polarized membrane localization of Cdc42 in response to CCL19 treatment.

Involvement of Cdc42 in CCL19-induced actin polymerization. Reorganization of the actin cytoskeleton is an early event in the migratory response to chemokines (19). To investigate whether actin cytoskeleton is reorganized in SCCHN cells stimulated by CCL19, we examined the F-actin staining using TRITC-labeled phalloidin. Treatment of CCL19 induced lamellipodia protrusion and cortical actin formation in the leading edge of SCCHN cell line PCI-4B (Fig. 3a) and PCI-37B (Fig. 3b). The changes in the actin cytoskeleton induced by CCL19 were largely abolished by CCR7 mAb, LY294002 or Toxin B, indicating the involvement of CCR7$\mathrm{PI} 3 \mathrm{~K}$ and possibly Cdc42 for the cystoskeletal changes. To further confirm whether $\mathrm{Cdc} 42$ is involved in the process, we knocked down Cdc42 in PCI-37B cells using siRNA against Cdc42, which resulted in over $90 \%$ knockdown of Cdc42 as indicated by Western blot analysis (Fig. 3c). Indeed knockdown of $\mathrm{Cdc} 42$ abolished the actin cytoskeletal changes in response to CCL19 treatment (Fig. 3a and b).

Cdc42 is required for activation of Rac induced by CCL19 stimulation. Lamellipodia formation in the leading edge is known to be mediated by $\operatorname{Rac}(12,14,20)$. So we investigated the level of activated Rac and its relationship with $\mathrm{Cdc} 42$. Similar to the changes of $\mathrm{Cdc} 42$, the level of activated Rac was found to increase significantly $0.5 \mathrm{~h}$ after CCL19 treatment as measured by the Rac GTPase assay (Fig. 4). In contrast, siRNA knockdown of Cdc42 efficiently inhibited the CCL19-induced activation of Rac (Fig. 4). These results indicate that $\mathrm{Cdc} 42$ is required for the activation of Rac induced by CCL19 in SCCHN.

CCR7 induces PI3K-mediated invasion and migration of metastatic SCCHN cell lines through Cdc42. Finally we determined whether $\mathrm{Cdc} 42$ is involved in the migration and invasion of SCCHN cells in response to CCL19. For this purpose, transwell migration and invasion assays were performed to analyze the role of $\mathrm{Cdc} 42$ on the capability of SCCHN cell lines to migrate and invade in response to CCL19. The presence of CCL19 in the lower chamber stimulated over 2-fold increase in the migration (Fig. 5) and invasion (Fig. 6) of PCI-4B and PCI-37B cells across the filter membrane. The increased cell migration (Fig. 5) and invasion (Fig. 6) induced by CCL19 were abolished by CCR7 mAb, LY294002, Toxin B or Cdc42 siRNA $(\mathrm{P}<0.01)$. These findings indicate that $\mathrm{Cdc} 42$ plays a key role in the CCL19induced chemotactic migration and invasion of SCCHN cells.

\section{Discussion}

Chemokines interact with G-protein-coupled receptors (GPCRs) and direct 'homing' of circulating haematopoietic cells and tumor cells can also adopt this strategy during the process of metastasis (2). As a GPCR, CCR7 has been shown to induce chemotaxis and invasion of SCCHN cells via activation of PI3K $(5,6,9)$. However, how CCR7-PI3K stimulates migration of SCCHN cells remains poorly understood. Here, we identified $\mathrm{Cdc} 42$ as an important downstream molecule of CCR7-PI3K pathway for polarized migration of SCCHN cells.

Results from immunohistochemical studys of 78 clinical cases showed that CCR7 and Cdc42 were highly expressed in SCCHN tumor tissues, and metastatic lymph nodes. The expression was significantly correlated with cervical lymph node metastasis and clinical stage, but was at a low level or absent in normal lymph nodes and oral mucosal tissues. CCR7 was reported to be a novel prediction biomarker of metastasis in cancer (7). Future studies including the immunohistochemical analysis of both CCR7 and Cdc42 might be useful to predict lymph node metastasis.

Our results showed that stimulation of CCL19 resulted in an elevated level of activated membrane-bound $\mathrm{Cdc} 42$ and concentration of $\mathrm{Cdc} 42$ in the leading edge of migrating 
a
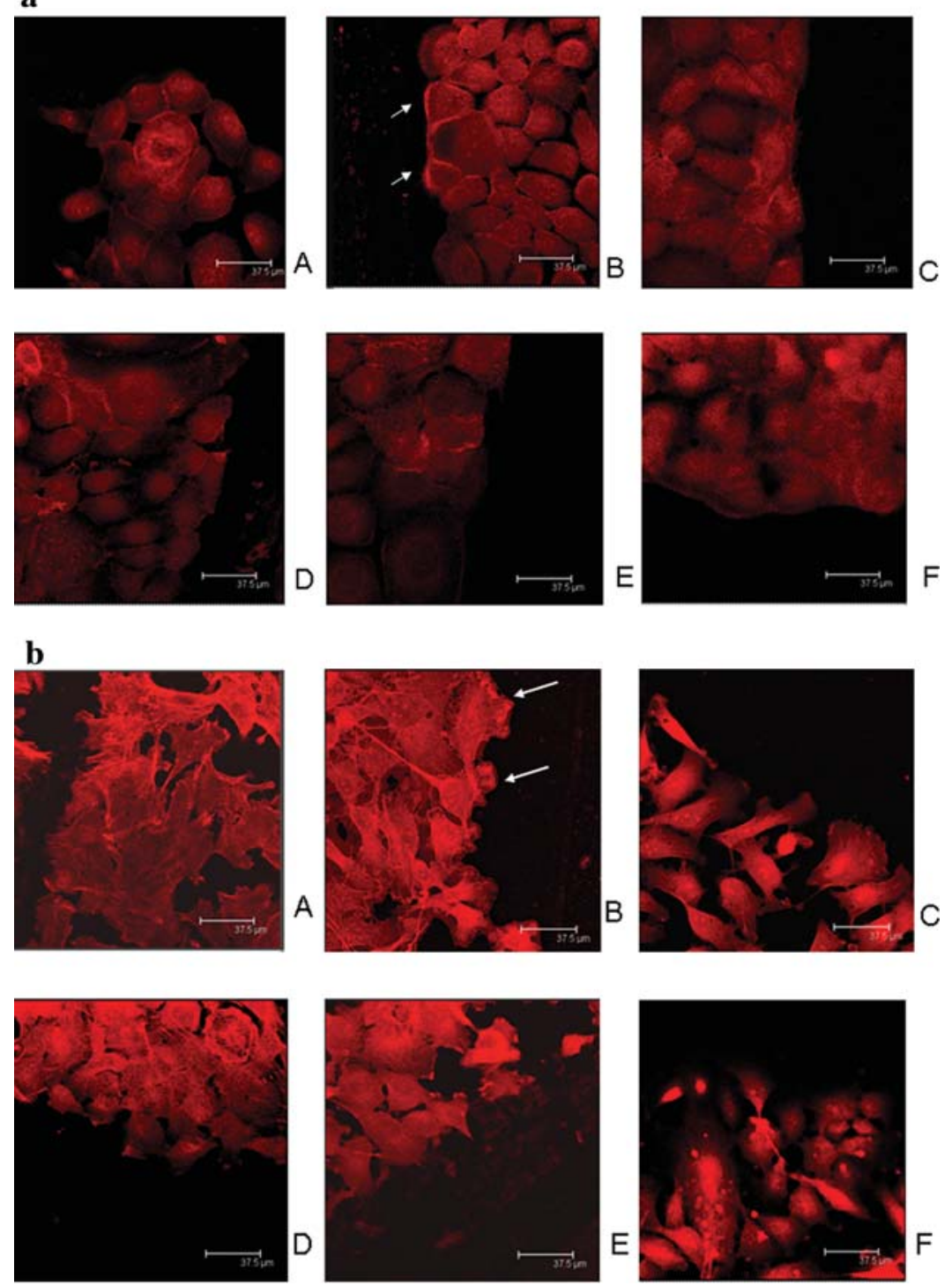

c

WCL

\section{$\operatorname{Cdc} 42$}

\section{$\beta$-actin}

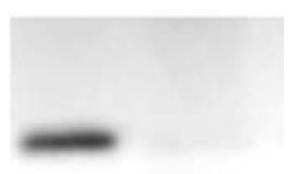

\section{Non-specific $\quad \mathrm{Cdc} 42$ \\ control siRNA siRNA}

Figure 3. Activation of Cdc42 is involved in CCL19-induced actin polymerization. (a) Characteristic organization of filamentous actin (F-actin) in PCI-4B and intense F-actin stain is visible in the leading edge after CCL19 stimulation (arrow). The change was blocked by pretreatment with inhibitors and Cdc42 siRNA. (A), control; (B), CCL19; (C), pretreated with CCR7 mAb and incubated with CCL19; (D), pretreated with LY294002 and incubated with CCL19; (E), pretreated with Toxin B and incubated with CCL19; (F), Cdc42 siRNA and incubated with CCL19. F-actin was stained with TRITC-labeled phalloidin and visualized using CLSM. (b) Characteristic organization of filamentous actin (F-actin) in PCI-37B. Intense F-actin stain is visible in the leading edge after CCL19 stimulation (arrow). The change was blocked by pretreatment with inhibitors and Cdc42 siRNA. (A), control; (B), CCL19; (C), pretreated with CCR7 mAb and incubated with CCL19; (D), pretreated with LY294002 and incubated with CCL19; (E), pretreated with Toxin B and incubated with CCL19; (F), Cdc42 siRNA and incubated with CCL19. F-actin was stained with TRITC-labeled phalloidin and visualized using CLSM. (c) SiRNA resulted in over 90\% knock down of Cdc42 in PCI-37B. SCCHN cells were transfected using 100 pmol Cdc42-specific or controled siRNA for 48 h. Levels of total Cdc42 were determined for whole cell lysates (WCL) were detected by Western blotting. Western blot analysis of ß-actin was done to confirm equal protein loading. 


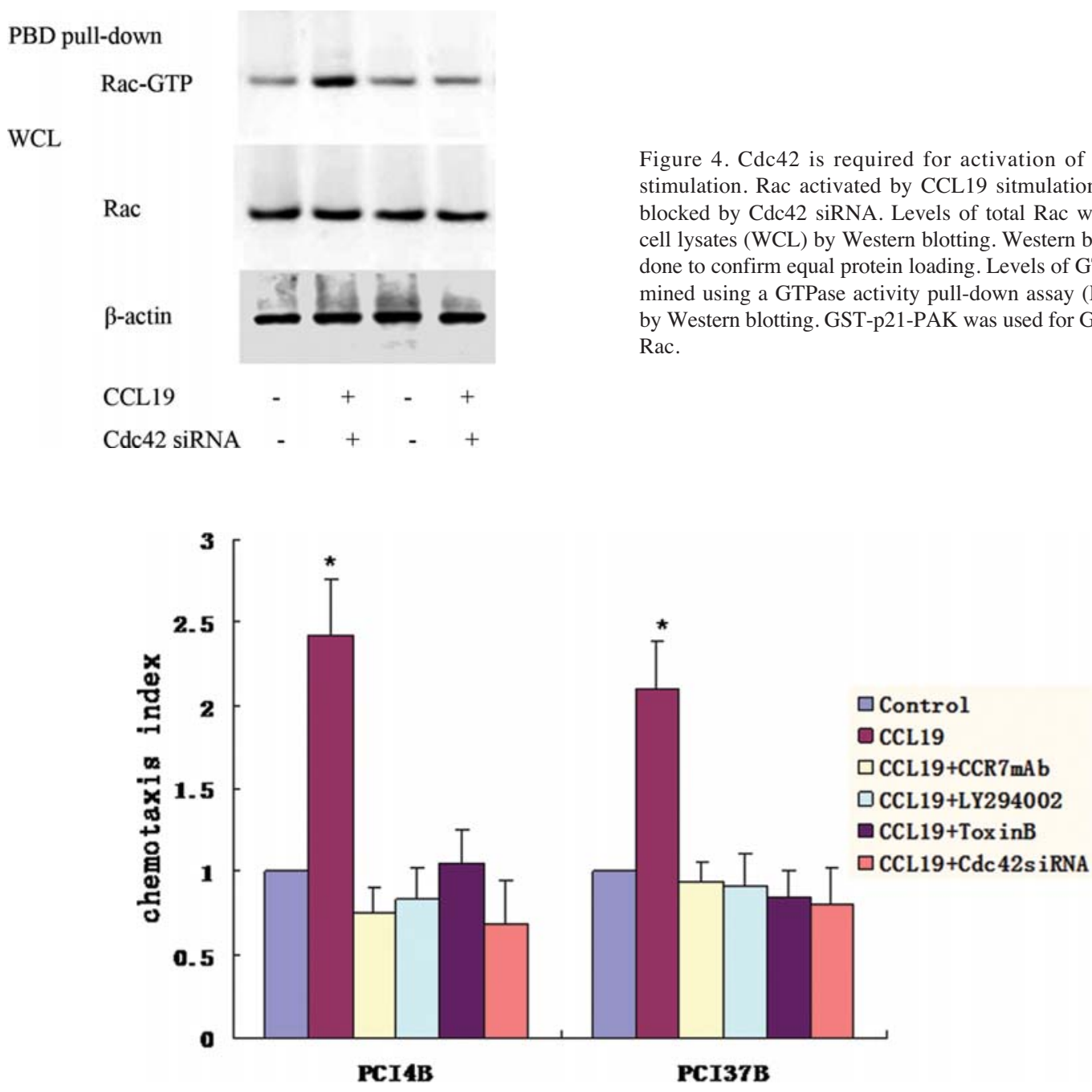

Figure 5. CCR7 induced PI3K-mediated migration of metastatic SCCHN cell lines through Cdc42. Cell migration was significantly increased by CCL19 stimulation and blocked by CCR7 siRNA (P<0.01). PCI-4B and PCI-37B were pretreated with/without CCR7 mAb, PI3K inhibitor LY294002, Toxin B and Cdc42 siRNA. CCL19 was the chemoattractant. Transwell inserts were used to analyze migration at $24 \mathrm{~h}$ post seeding. The chemotaxis index was expressed as percentage of control sample which was not exposed to CCL19.

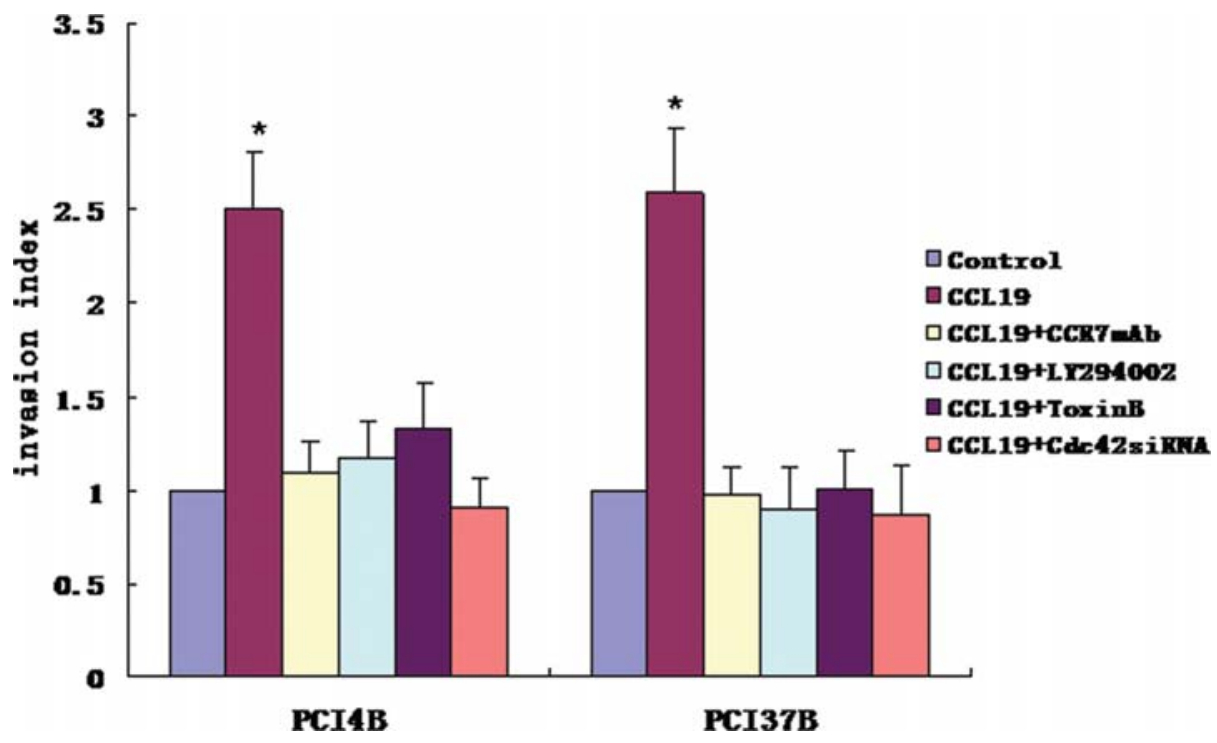

Figure 6. CCR7 induces PI3K-mediated invasion of metastatic SCCHN cell lines through Cde42. Cell invasion was significantly increased by CCL19 stimulation and blocked by CCR7 siRNA (P<0.01). PCI-4B and PCI-37B were pretreated with/without CCR7 mAb, PI3K inhibitor LY294002, Toxin B and Cdc42 siRNA. CCL19 was the chemoattractant. Matrigel-coated inserts were used to analyze invasion at $48 \mathrm{~h}$ post seeding. The invasion index was expressed as percentage of control sample which was not exposed to CCL19. 
SCCHN cells in a CCR7- and PI3K-dependent manner. Rho guanosine triphosphatese (GTPase) proteins act as molecular switches to control cellular processes by cycling between active (GTP-bound) and inactive (GDP-bound) states $(14,18,21)$. Rho, Rac and Cdc42 are known to mediate the formation of stress fiber, lamellipodia and filopodia, respectively $(12,14)$. Rho GTPase function is critically dependent on association of the GTPases with membranes and their subcellular locations (22). It is well-established that activated GTPases translocate to the cell membrane, where they bind and activate their downstream effectors $(17,18)$. In several previous studies, micropipette assays using microinjection system $(10,11,19)$ were carried out to study cell polarity. In this study, we examined cellular localization of Cdc42 using wound-healing assays $(23,24)$. Consistent with previous studies, the accumulation of $\mathrm{Cdc} 42$ in the leading edge provides further support for the activation and role of $\mathrm{Cdc} 42$ in SCCHN cells. The increased activation level and polarized membrane localization of $\mathrm{Cdc} 42$ were diminished by CCR7 and PI3K inhibition, which indicated that CCL19 stimulation leads to activation of Cdc42 via the CCR7-PI3K pathway in SCCHN cells. Because wound healing assay is a static study, we still need microinjection and chemokine gradient studies to further explore the time-lapse dynamic changes.

CCL19 induces the polarized actin polymerization and the protrusion of lamellipodia in SCCHN cells. In contrast, the CCL19-stimulated formation of lamellipodia was abolished by Toxin B and Cdc42 siRNA, implicating that Cdc42 might be functional upstream of Rac in SCCHN cells. Indeed, siRNA knockdown of Cdc42 decreased the level of activated Rac in PCI-37B cells, which confirm that Cdc42 is required for Rac activation. Cdc 42 has been shown to be a strong activator of Rac $(21,25,26)$ and Rho $(27)$ in many cell types. For example, IRSp53 (21) binding to Cdc42 acts as a direct link between $\mathrm{Cdc} 42$ and Rac so that $\mathrm{Cdc} 42$ can induce Rac-dependent lamellipodium extension. In addition, $\mathrm{Cdc} 42$ has been reported to activate the serine/threonine kinase MRCK, which can promote myosin phosphorylation (27). A hierarchy among these GTPases was established, in which activated Cdc 42 activates Rac, which in its turn activates RhoA (12).

Although Cdc42 is well known to control filopodial formation, we rarely find fipodia in SCCHN cells upon CCL-19 stimulation. It is difficult to observe filopodia in SCCHN cells unless Rac activity is first inhibited (25). Possibly activation of Cdc42 leads to rapid activation of Rac and lamellipodial extension, which prevents the detection of filopodia.

Consistent with its effect on polarity of the actin cytoskeleton, $\mathrm{Cdc} 42$ plays a critical role in polarized migration of SCCHN cells. Toxin B and Cdc42 siRNA significantly inhibited the chemotactic migration and invasion of SCCHN cells. These findings together support the idea that $\mathrm{Cdc} 42$ might plays a central role in CCL19-induced PI3K-mediated polarity of actin cytoskeleton and chemotaxis of SCCHN cells.

Metastatic disease is the major barrier to curative treatments, and it is necessary to decode this aspect of cancer behavior if we are to develop more effective and less toxic therapies (2). Signaling pathways controlling directional cell migration are not linear, but integrate signals from a plethora of upstream switches into a molecular matrix, resulting in complex cellular responses. Further work is required to elucidate the sequence of events leading to the CCR7mediated metastatic phenotype, which will enable the development of therapeutic strategies aiming at blocking these carcinogenic and metastatic effects.

\section{Acknowledgements}

We would like to thank Dr Schwartz M.A. (University of Virginia) for supplying GST-PBD vector and thank Jie Liu, Chun-yan Li for their excellent technical assistance in cell culture and siRNA experiments, respectively. This research was supported by grant from the National Natural Science Foundation of China (No. 30672331).

\section{References}

1. Greenlee RT, Hill-Harmon MB, Murray T and Thun M: Cancer Statistics, 2001. CA Cancer J Clin 51: 15-36, 2001.

2. Eccles SA: Targeting key steps in metastatic tumour progression. Curr Opin Genet Dev 15: 77-86, 2005.

3. Zlotnik A and Yoshie O: Chemokines: a new classification system and their role in immunity. Immunity 12: 121-127, 2000.

4. Zlotnik A: Chemokines in neoplastic progression. Semin Cancer Biol 14: 181-185, 2004.

5. Wang J, Xi L, Hunt JL, et al: Expression pattern of chemokine receptor 6 (CCR6) and CCR7 in squamous cell carcinoma of the head and neck identifies a novel metastatic phenotype. Cancer Res 64: 1861-1866, 2004.

6. Wang J, Zhang X, Thomas SM, et al: Chemokine receptor 7 activates phosphoinositide-3 kinase-mediated invasive and prosurvival pathways in head and neck cancer cells independent of EGFR. Oncogene 24: 5897-5904, 2005.

7. Wang J, Seethala RR, Zhang Q, et al: Autocrine and paracrine chemokine receptor 7 activation in head and neck cancer: implications for therapy. J Nat Cancer Inst 100: 502-512, 2008.

8. Liu F, Zhao Z, Li P, Ding X, Zong Z and Sun C: Mammalian target of rapamycin (mTOR) is involved in the survival of cells mediated by chemokine receptor 7 through PI3K/Akt in metastatic squamous cell carcinoma of the head and neck. Br J Oral Maxillofac Surg 48: 291-296, 2010.

9. Zhao Z, Liu F and Sun C: Effect of chemokine reportor 7 small interfering RNA on proliferation and invation of squamous cell carcinoma of head and neck. Zhonghua Kou Qiang Yi Xue Za Zhi 44: 5-10, 2009.

10. Janetopoulos C, Ma L, Devreotes PN and Iglesias PA: Chemoattractant-induced phosphatidylinositol 3,4,5-trisphosphate accumulation is spatially amplified and adapts, independent of the actin cytoskeleton. Proc Natl Acad Sci USA 101: 8951-8956, 2004.

11. Xu J, Wang F, Keymeulen AV, et al: Divergent signals and cytoskeletal assemblies regulate self-organizing polarity in neutrophils. Cell 114: 201-214, 2003.

12. Hall A: Rho GTPases and the actin cytoskeleton. Science 279: 509-514, 1998.

13. Yanagawa Y and Onoé K: CCL19 induces rapid dendritic extension of murine dendritic cells. Blood 100: 1948-1956, 2002.

14. Etienne-Manneville S and Hall A: Rho GTPases in cell biology. Nature 420: 629-635, 2002.

15. Yanagawa Y and Onoé K: CCR7 ligands induce rapid endocytosis in mature dendritic cells with concomitant up-regulation of Cdc42 and Rac activities. Blood 101: 4923-4929, 2003.

16. Ueda H, Morishita R, Yamauchi J, Itoh H, Kato K and Asano T: Regulation of Rac and Cdc42 pathways by Gi during lysophosphatidic acid-induced cell spreading. J Biol Chem 276: 6846-6852, 2001.

17. Del Pozo MA, Price LS, Alderson NB, Ren XD and Schwartz MA: Adhesion to the extracellular matrix regulates the coupling of the small GTPase Rac to its effector PAK. EMBO J 19: 2008-2014, 2000. 
18. Buchsbaum RJ: Rho activation at a glance. J Cell Sci 120: 1149-1152, 2007.

19. Muller A, Homey B, Soto H, et al: Involvement of chemokine receptors in breast cancer metastasis. Nature 410: 50-56, 2001.

20. Evers EE, Zondag GC, Malliri A, et al: Rho family proteins in cell adhesion and cell migration. Eur J Cancer 36: 1269-1274, 2000.

21. Ridley AJ: Rho GTPases and cell migration. J Cell Sci 114: 2713-2722, 2001.

22. Wennerberg K and Der CJ: Rho-family GTPases: it's not only Rac and Rho (and I like it). J Cell Sci 117: 1301-1312, 2004.

23. Nikolic DL, Boettiger AN, Bar-Sagi D, Carbeck JD and Shvartsman SY: Role of boundary conditions in an experimental model of epithelial wound healing. Am J Physiol Cell Physiol 291: C68-C75, 2006.
24. Keese CR, Wegener J, Walker SR and Giaever I: Electrical wound-healing assay for cells in vitro. Proc Natl Acad Sci USA 101: 1554-1559, 2004.

25. Mackay DJG and Hall A: Rho GTPases. J Biol Chem 273: 20685-20688, 1998.

26. Hirsch DS, Shen Y and Wu WJ: Growth and motility inhibition of breast cancer cells by epidermal growth factor receptor degradation is correlated with inactivation of $\mathrm{Cdc} 42$. Cancer Res 66: 3523-3530, 2006.

27. Schwartz M: Rho signalling at a glance. J Cell Sci 117: 5457-5458, 2004. 